\title{
Representaciones sociales de docentes de una universidad chilena acerca de la escri- tura académica y de su enseñanza-aprendizaje
}

\author{
Social representations of lecturers at a Chilean university regarding academic writing \\ and its teaching and learning
}

\author{
María Verónica Sánchez Gibbons \\ e-mail: mvsanche@uc.cl \\ Pontificia Universidad Católica de Chile. Chile
}

\section{Resumen}

En el contexto del desafío que representa para los estudiantes incorporarse a las prácticas de escritura académica y al rol que el profesorado tiene en dicho proceso, el presente estudio de naturaleza exploratoria indaga en las representaciones sociales de docentes de diversas disciplinas de una universidad chilena acerca de la escritura académica y de su enseñanza-aprendizaje, con el fin de obtener información clave para el diseño de intervenciones efectivas que propicien el desarrollo de esta habilidad por parte de los y las estudiantes. Para este objetivo se realizaron varios grupos de discusión, que fueron analizados a partir de un análisis cualitativo de contenido. Entre los resultados más destacados se encuentra el acuerdo entre los docentes sobre la relevancia de que los estudiantes desarrollen habilidades de escritura académica y la necesidad de que exista una instancia, fuera del contexto de las cátedras que ellos tienen a cargo, de trabajo de esta herramienta. Por otra parte, en cuanto a las estrategias de enseñanza y aprendizaje de los profesores, se revela la falta de precisión de los docentes con respecto a qué es escribir académicamente y la consideración de aspectos como la motivación y la práctica para la enseñanza-aprendizaje de esta habilidad.

Palabras clave: alfabetización; competencias de escritura; competencias específicas de una disciplina; educación superior; enseñanza y aprendizaje; profesor.

\section{Abstract}

In the context of the challenge that students face to incorporate the practices of academic writing and the role that teachers have in this process, this exploratory research enquires into social representations of lecturers of diverse disciplines from a Chilean university regarding academic writing and its teaching and learning, in order to obtain key information for the design of effective interventions that foster this skill's development by students. To this objective, data were produced from discussion groups and then analysed through a qualitative content analysis. One of the most outstanding results is the agreement among teachers of the relevance of the development of academic writing skills by students and the need for an instance, outside the context of the chairs they are in charge of, to work to this aim. On the other hand, regarding teachers' teaching and learning strategies, results show a lack of accuracy in the meaning of academic writing for lecturers and their consideration of aspects such as motivation and practice for teaching and learning this skill. (up to 250 words).

Keywords: literacy; written skills; subject-specific competences; higher education; teaching and learning; teacher.

Recibido / Received: 14-01-2020

Aceptado / Accepted: 26-05-2020

Publicación en linea / Published online: 01-07-2020

Cómo referenciar este artículo / How to reference this article:

Sánchez Gibbons, M. V. (2020). Representaciones sociales de docentes de una universidad chilena acerca de la escritura académica y de su enseñanza-aprendizaje. Tendencias Pedagógicas, 36, pp. 44-58. doi: 10.15366/tp2020.36.04 


\section{Introducción}

Tanto el conocer como el hacer de cada una de las disciplinas del conocimiento se encuentra estrechamente vinculado a una forma específica y especializada de escribir (Carter, 2007) por lo que los estudiantes de la educación superior, para transitar exitosamente en su formación universitaria y convertirse en miembros de una comunidad de conocimiento especializado, tienen el reto de insertarse en una comunidad discursiva disciplinar (Swales, 1990).

Por otra parte, dicho desafío estaría intervenido por la necesidad de aprender a enfrentar la escritura desde una nueva forma de operar que requiere manejar ciertas estrategias cognitivas para alcanzar un nivel avanzado de escritura, lo que constituye un reto importante para la cognición humana (Ke$\operatorname{logg} \&$ Whiteford, 2009). Para ello, desde la perspectiva cognitiva, se propone un alto nivel de práctica de modo de que el alumno-escritor genere control sobre los procesos cognitivos, motores y perceptuales involucrados en el proceso de escritura (Kellogg \& Whiteford, 2009) y reduzca las demandas cognitivas involucradas (McCutchen, 1988), sobre todo en los procesos individuales de alto nivel como la planificación de ideas o las operaciones de revisión, entre otras (Kellogg, 1994). El manejo de estas estrategias jugaría un rol crucial en la diferenciación de escritores noveles de escritores expertos (Hayes \& Flower, 1980; Flower, Hayes, Carey, Schriver \& Stratman, 1986), como también resultaría altamente influyente el nivel de conocimiento disciplinar que el escritor maneja (Alexander, 1997; Kellogg \& Whiteford, 2009).

Para lograr lo anterior, una de las propuestas que entrega la psicología cognitiva radica en la práctica deliberada (deliberate practice), la que involucra la práctica dirigida a mejorar una meta específica. Esto requiere que el aprendiz se involucre de forma importante en actividades cuidadosamente diseñadas por un instructor y que reciba retroalimentación sobre su desempeño (Ericsson, 2006; Kellog \& Whiteford, 2009).

Pese a lo altamente demandante del desarrollo de esta habilidad en el contexto académico-disciplinar y el importante rol que cumplen en este proceso los docentes, la evidencia indica que estos tenderían a no dar instrucciones explícitas sobre este aspecto (Stagnaro y Chosco, 2013). Esto se condice con estudios que indican que el valor agregado del currículo universitario con respecto al desarrollo de habilidades de escritura académica es muy bajo (Curry \& Hager, 1987; White, 1989; Miño, Hugo \& Sánchez, 2012). La variabilidad de estos resultados, según los diferentes contextos institucionales nacionales e internacionales, así como su falta de especificidad, pone de manifiesto la necesidad de indagar y reconocer las comprensiones que los y las docentes tienen de la escritura académica y de su enseñanza-aprendizaje en cada contexto.

En este escenario, el presente estudio tiene como propósito explorar las representaciones sociales (Farr \& Moscovici, 1984; Moscovici, 2003; Marková, 1996; Wagner et al., 1999) de docentes de diversas disciplinas de una universidad chilena con respecto a su comprensión de la escritura académica y a su enseñanza-aprendizaje. El poder obtener y analizar información al respecto resulta pertinente dada la íntima relación entre las representaciones sociales de la escritura académica y de su enseñanzaaprendizaje y las estrategias que los profesores emprenden con sus estudiantes (Hernández, 2012).

$\mathrm{El}$ interés de este estudio, entonces, es indagar en la dimensión social de la enseñanza-aprendizaje de la escritura académica y de su conceptualización debido a su relevancia para estos procesos, lo que está conceptualizado y comprendido desde el marco epistémico relacional en el que el individuo y lo colectivo, la naturaleza y la cultura están en una constante interacción en el contexto de la construcción de estructuras de conocimiento. En este marco, las representaciones sociales funcionan como un contorno que da forma y delimita a estas últimas (Castorina, 2010).

Las representaciones sociales (en adelante RS), que conllevan una relación de interdependencia entre lo social y lo individual, son utilizadas tanto por grupos como por individuos para comunicarse y actuar (Moscovici, 1963) y, a la vez, son construidas socialmente a partir de la conversación y acción (Wagner et al., 1999).

En el caso del campo educativo, el estudio de las RS resulta de especial relevancia, pues estas afectan la trayectoria formativa de los estudiantes, ya sea a partir de los conceptos y expectativas de los docentes como también de las de los propios alumnos (Castorina \& Kaplan, 1995, 1997). Pese a la relevancia del constructo de las RS para la comprensión de la enseñanza-aprendizaje de la escritura académica en el contexto universitario, existen a la fecha pocos estudios al respecto ya que la investigación de este campo tiende a concentrarse en las RS de escritura en los niveles de educación básica y media (Ortiz, 2011). Por otra parte, las investigaciones existentes exploran con más frecuencia las 
RS de los estudiantes universitarios que las de los docentes, como es el caso del estudio de Roux (2008).

En cuanto a los estudios publicados que tienen como objeto las RS de docentes y/o estudiantes universitarios acerca de la escritura académica y/o la enseñanza-aprendizaje de la misma se pueden mencionar las investigaciones de Natale (2011); Marincovich y Salazar (2011); López y Pedraza (2012); Stagnaro y Chosco (2013); Corcelles, Oliva, Castelló y Milian (2015); Castelló y Mateos (2015); Ortiz (2015) y Marincovich, Velásquez, Córdova y Cid (2016).

Entre los principales resultados relativos a las RS sobre la escritura académica se encuentran los desajustes entre las expectativas de docentes y estudiantes en cuanto al desempeño esperado en el campo de la escritura académica (Stagnaro y Chosco, 2013). A esto se suma que las actividades de escritura habituales en el contexto universitario tienen mayor relación con la demostración del conocimiento disciplinar de los alumnos que con la transformación del conocimiento (Natale, 2011; Corcelles et al., 2015; López \& Pedraza, 2012) y a que existirían, por parte de docentes y estudiantes, dos orientaciones principales con respecto a la escritura académica: la prescriptiva y la formativa (Marincovich et al., 2016).

Por otra parte, los principales resultados relativos a las RS sobre la enseñanza-aprendizaje de la escritura académica evidencian que los docentes no se consideran responsables de dicho proceso formativo en el contexto de sus cátedras (López \& Pedraza, 2012). A lo anterior se suma evidencia que indica que no se explicitan las expectativas de logro a los estudiantes a partir de la entrega de instrucciones y/o instrumentos de evaluación (López \& Pedraza, 2012), la ausencia del trabajo de la escritura como proceso en la acción didáctica de los docentes (Ortiz, 2015) y que los profesores expresan no saber qué exigir a sus estudiantes ni cómo gestionar la enseñanza-aprendizaje de la escritura académica (Natale, 2011).

En cuanto a los métodos predominantemente utilizados en estos estudios se encuentran la realización de encuestas individuales y el análisis de consignas (Stagnaro \& Chosco, 2013), la aplicación de cuestionarios (Corcelles et al., 2015; Castelló \& Mateos, 2015), la realización de entrevistas semiestructuradas en profundidad y grupos focales (Marincovich \& Salazar, 2011; Natale, 2011; Marincovich et al., 2016; Ortiz, 2015) y la observación de clases (Ortiz, 2015). Es así como en gran parte de estas investigaciones las técnicas de producción de datos empleadas se corresponden más con el levantamiento de información a partir del discurso y las experiencias de los sujetos como individuos más que de una voz colectiva, lo que no sería consistente con el constructo de las RS, diagnóstico que coincide con el de Ortiz (2011).

Por último, es necesario señalar que los estudios que exploran las RS de los docentes universitarios de la escritura académica y/o su enseñanza-aprendizaje tienden a concentrarse en un solo campo disciplinar, como es el caso de los estudios de Marincovich y Salazar (2011) y López y Pedraza (2012). Es así como fuera del caso del estudio conducido por Marincovich et al. (2016), que considera en su muestra a estudiantes y docentes de cinco carreras, existe una ausencia de investigaciones que indaguen en los consensos entre docentes pertenecientes a diferentes campos disciplinares.

En este marco, cabe indicar que, pese al valor innegable de estos hallazgos, existe la necesidad de seguir indagando en las RS de los docentes universitarios acerca de la escritura académica y su enseñanza-aprendizaje desde una perspectiva que trabaje este constructo en concordancia con la teoría al respecto y que considere las múltiples realidades disciplinares que coexisten en el contexto universitario.

\section{Metodología}

Con el objeto de abordar el propósito de la presente investigación de naturaleza exploratoria, se adoptó un enfoque cualitativo desde el marco socioconstructivista (Creswell, 2007). En cuanto al campo seleccionado (Creswell, 2007), este correspondió a la Pontificia Universidad Católica de Chile. Esta elección se sustenta en que se cuenta con datos, previos a este estudio, acerca del bajo nivel de apoyo que los estudiantes perciben que sus docentes les entregan en relación a su incorporación a la escritura académica disciplinar (Miño, Hugo \& Sánchez, 2012). 


\subsection{Producción de datos}

Dado que en el caso de las RS el entorno simbólico «se re-construye a través de las actividades de los individuos, sobre todo por medio del lenguaje» (Marková, 1996), la técnica seleccionada fue la realización de grupos de discusión (Canales, 2006). Cabe indicar que la elección de esta técnica resulta coherente con el constructo a estudiar ya que esta permite vincular a los sujetos en una comunidad y levantar una palabra común (Canales, 2006).

Como estímulo para la conversación se enfrentó a cada uno de los grupos a la resolución de dos tareas, para lo que contaron con una hora y media de tiempo. La primera de ellas consistió en responder grupalmente a la pregunta «¿Qué necesitan los estudiantes para escribir de forma adecuada en el contexto académico?» y la segunda en generar un modus operandi o recetario de qué debe hacerse para aprender a escribir académicamente. Cada grupo pudo organizar sus respuestas de forma libre, contando para ello con lápices, post-it y papelógrafos.

\subsection{Muestra}

En relación al proceso de muestreo, se utilizó uno de tipo no probabilístico, ya que la elección de los informantes clave se determinó a partir de la naturaleza de la investigación. Específicamente, se utilizó el muestreo de casos-tipo, ya que los grupos de discusión se constituyeron a partir de ciertas características de los informantes clave (Hernández, Fernández y Baptista, 2010). De esta manera se convocó a un total de 55 docentes de la casa de estudios indicada, pertenecientes a diferentes disciplinas (Enfermería, Arte, Historia, Sociología, Química, Teología, Educación, Agronomía e Ingeniería Forestal, Odontología, Construcción Civil, Letras, Medicina, Trabajo Social, Música, Derecho, Matemáticas, Comunicaciones, Economía, Estética, Teatro, Diseño, Biología y Física) que cumplieran con desempeñarse como miembros de planta ordinaria o adjunta de una facultad realizando labores de docencia en pregrado y que no tuvieran relación previa de trabajo con la investigadora principal.

La naturaleza exploratoria del presente estudio involucró la formación de tres grupos de discusión, número que resulta coherente con las recomendaciones de conformación de grupos para el levantamiento de RS (Wagner et al., 1999). El primero de ellos estuvo integrado por tres docentes (Odontología, Química, Teología), el segundo por cuatro docentes (Enfermería, Educación, Construcción Civil y Odontología) y el tercero por cuatro docentes (Educación, Enfermería, Arte y Sociología). De esta manera se cumplió con generar instancias de diálogo entre diferentes disciplinas sobre el fenómeno en cuestión.

La participación en el proyecto fue explicada en detalle a los informantes clave, quienes accedieron a contribuir en él a partir de la firma de un consentimiento informado que sigue los protocolos de investigación de la Pontificia Universidad Católica de Chile.

\subsection{Análisis de los datos}

En concordancia con la naturaleza del producto de los grupos de discusión, así como la de las RS, el análisis de la información producida se realizó a partir de las conceptualizaciones conjuntas de los docentes y no de sus concepciones individuales. De esta manera el foco del análisis estuvo puesto en las interacciones entre los sujetos y sus consensos sobre los fenómenos estudiados, con un énfasis en el proceso mismo de conceptualización durante el desarrollo de la discusión (Larraín \& Haye, 2014). Para lo anterior, las instancias de discusión fueron transcritas y analizadas por medio de un análisis de contenido cualitativo (Bardin, 1996) a partir del desarrollo de categorías inductivas a partir de los procesos de codificación inicial o en primer plano (Coleman \& Unrau, 2005) y de codificación central o en segundo plano (Grinnell, 1997). A partir de la primera codificación se identificaron las categorías, se evaluaron las unidades de análisis y se asignaron códigos; en la segunda codificación los códigos 
ya construidos se agruparon en categorías mayores y a la vez en temas. El proceso antes descrito se apoyó en la utilización del software de análisis cualitativo Atlas Ti.

Por último, es relevante dar cuenta de que el rol que la investigadora adoptó en este estudio correspondió a uno de naturaleza de participación activa, papel en el que no solo fue observadora, sino también una participante moderada (Hernández, Fernández \& Baptista, 2010).

\section{Resultados}

A continuación, se presenta la sistematización de los resultados obtenidos a partir de los grupos de discusión realizados. Por un lado, se da cuenta de las RS de los docentes en torno a la escritura académica y, por otro lado, de las RS relativas a su enseñanza-aprendizaje.

\subsection{Representaciones sociales acerca de la escritura académica}

A continuación, se presenta lo indicado por los docentes en relación a aquello que involucraría un manejo adecuado de la escritura académica, lo que permite identificar su comprensión de este constructo desde su perspectiva. Esta se encuentra representada en cuatro categorías:

\section{1.a. Manejo adecuado de las convenciones formales y del conocimiento disciplinar}

Escribir académicamente se caracterizaría por el manejo adecuado de ciertas convenciones formales de los escritos, tales como la estructura de los géneros escritos que se debe producir en este contexto y el uso de los mecanismos de citación. Este tipo de escritura involucraría un manejo profundo del lenguaje y del campo de conocimiento de la disciplina desde la que se está generando la escritura. Esta caracterización se evidencia en el siguiente diálogo entre una profesora de Química, un profesor de Teología y una profesora de Odontología:

«Profesora de Química: Ya, que más, mira, puse dentro de, para escribir académicamente, como, qué tenemos que hacer: conocer los fundamentos de la disciplina, hay que tener flexibilidad de pensamiento y se transmite, enseña y aprende con el ejemplo que es lo mismo, sí, en el fondo, se adquiere con la madurez. Qué otra cosa podríamos poner...

Profesor de Teología: Pusiste lo-lo, las cosas ya más formales, como conocimiento de la disciplina del lenguaje...

Profesora de Odontología: Sí, eso está.

Profesora de Química: Eso que debe conocer profundamente la disciplina y su lenguaje.

Profesor de Teología: Ah, ya. Y su lenguaje, sí. También las convenciones de...

Profesora de Química: Sí, o sea... ¿̇ah?

Profesor de Teología: Las convenciones así de transmisión, o sea, por ejemplo, no se po [sic], en mi área se espera un artículo que tenga una longitud determinada, te dai [sic] cuenta.

Profesora de Química: Ahhh, ya.

Profesora de Odontología: Los protocolos, una cosa así.»

Cabe indicar que, en las discusiones entre los docentes, se denota poca especificidad en relación a aquello que implicaría el manejo de las convenciones formales. Esto podría significar falta de metalenguaje para hacer referencia a aspectos vinculados a la naturaleza de los textos y la no conceptuali- 
zación de los escritos como géneros discursivos. La generalidad con la que se representan las características formales de los textos puede evidenciarse en la siguiente discusión entre docentes de las áreas de las Ciencias de la Salud, las Ciencias Exactas y las Humanidades:

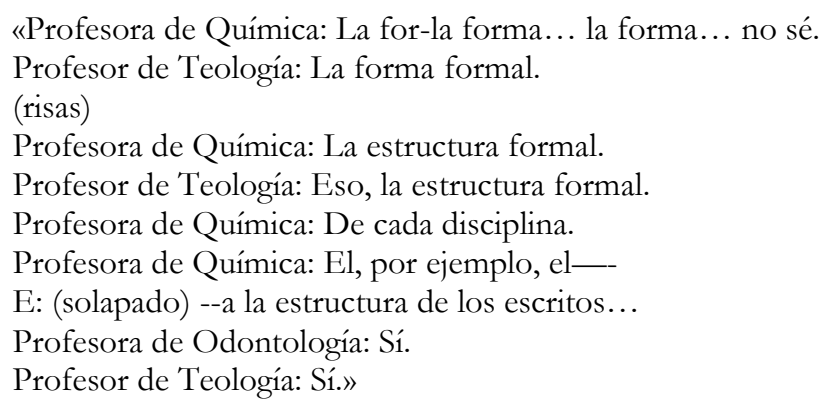

Frente a la indeterminación anterior, aparece la claridad y la relevancia que los docentes otorgan al manejo del tecnolecto disciplinar y del quehacer disciplinar, como pilares fundamentales de una adecuada escritura académica. Estos aspectos tendrían una primacía con respecto a las herramientas de redacción o de manejo de las convenciones del uso de la lengua escrita:

\begin{abstract}
«Claro, porque para escribir académicamente, por ejemplo, en cada disciplina, uno tiene que conocer muy bien la disciplina, y no confundir términos en la disciplina para transmitir bien, eh, el mensaje que uno quiere dar. Entonces para aprender a escribir académicamente uno tiene que previamente tener los conocimientos previos que deben ser profundos, claros y correctos... Sí, porque uno puede tener muy buena redacción, o muy buen vocabulario, pero si no entiendes lo que estás, lo que hiciste en el laboratorio en mi caso, ya, por qué paso eso, por qué la reacción ocurrió y en esta vez no ocurrió...» (Profesora de Química)
\end{abstract}

\title{
1.b. Alta situacionalidad con respecto al contexto disciplinar y a la cultura
}

Un segundo aspecto nuclear en las representaciones que los docentes tienen de este constructo tiene relación con su alta situacionalidad respecto al contexto disciplinar y cultural-temporal. Es así como resultaría complejo conceptualizar la escritura académica como un fenómeno estático e invariable según la realidad cultural:

\footnotetext{
«Claro, pero es que si tú dices la escritura académica es esto, tún, y es como si eso fuera hoy, y fue así hace cincuenta años atrás y va a ser así en doscientos años más, o sea, si tú crees que las cuestiones son así de rígidas en el fondo, no está atendiendo a la cultura; porque la cultura ha implicado que la escritura académica varíe muchísimo su forma, y va a seguir variando mucho más entonces hay una-un cierto nivel de flexibilidad que tenemos que tener nosotros y que tenemos que transmitir también...» (Profesor de Teología)
}

Además de referirse al factor temporal y cultural, los profesores hacen reiterados énfasis en las diferencias existentes en cómo se escribe y en qué se espera de la escritura en los diferentes contextos disciplinares. Dichas diferencias estarían íntimamente vinculadas con la naturaleza del conocimiento, así como también con las funciones sociales de cada disciplina:

«Pero, imagínate que en salud, o sea por otra parte, en salud, la parte de ciencias biológicas oo... medicina, tiene que ser súper eh... pragmáticos y decir bueno, la evidencia dice que, tal cosa, porque imagínate que, una enfermedad diga, mira pero, puede que sí, puede que no... aquí, pa [sic] acá, pa [sic] todos lados, eh... en salud uno quiere respuestas lo más precisas 
posibles porque es vida o muerte, entonces, como las distintas disciplinas hacen de que tengan un estilo.» (Profesora de Enfermería)

Estas diferencias no serían solo perceptibles en el ejercicio profesional, sino que marcarían la escritura de los estudiantes, específicamente en relación a cómo estos conciben la construcción de relaciones lógicas en sus textos:
«Profesora de Educación: ... estoy pensando en lo-en los estudiantes que... que van en el programa de formación pedagógica, vienen ya con una forma de escritura marcada por la disciplina de origen... y entonces luego se enfrentan a una forma de escritura que en educa- ción se les solicita y... donde... es más-como está vinculado a ciencias sociales, donde, el tema de la argumentación, un análisis como más de-desagregado y cualitativo de los fenóme- nos, y... entonces los que vienen de biología, de matemática-
Profesora de Arte: Les cuesta mucho eso.
Profesora de Educación: Claro, es otra forma de razonar, es otra forma de escribir. Y a los de arte también.
Profesora de Arte: Me imagino (risas).
Profesora de Educación: Porque yo creo que a lo-los de, yo creo que los de matemáticas y de ciencias, eh, sienten que tienen que-se les pide demasiada argumentación, demasiadas vueltas a-demasiado retórico para-porque ellos dicen dos o tres características, ya, esto es... Y a los de arte, también en el fondo, también como esquemati-hacerlo en, en esquema, yo creo que se sienten también restringidos. Nosotros, no, estamos acostumbrados a la reflexión como mucho más libre, dicen, más personal, lo mismo ahora que tú estabas planteando, entonces, eh...»

Estas diferencias dificultarían la concepción de la escritura en la universidad como un constructo único y transversal a los diferentes contextos disciplinares. De hecho, al enfrentarse al estímulo del grupo de discusión relativo a responder qué es la escritura académica, los profesores evidenciaron cierto nivel de conflicto:

«Profesora de Arte: Entonces yo lo interpreté un poco distinto, pero quizás es mejor ponerme, o sea como ponerse en el contexto de la universidad entera, y no en particular, por ejemplo, de los estudiantes que tienen esta, como los de literatura, los de arte, que en el fondo usan de manera quizás más creativa el lenguaje... que no necesariamente calza con una escritura académica, no sé. Es problemático eso, cómo podemos hacerlo...

Profesora de Educación: Yo creo que es importante la diferencia, ¿no? Sí, porque...

Profesor de Sociología: De todas maneras.»

Lo anterior pone en tensión la posibilidad de conceptualizar la actividad de escribir en y para el contexto académico como escritura académica y abre la necesidad de referirse a esta actividad como escritura disciplinar, al poner el énfasis de la misma en su origen en las diferentes áreas del conocimiento y las prácticas que cada una de ellas involucra. La necesidad de esta diferenciación se hace aún más evidente en las representaciones de los docentes cuando estos indican el riesgo de concebir la escritura académica como una realidad homogénea:

«Se va a perder esta delicadeza que tiene cada una de las profesiones, o sea, yo, pa mí, es escribir bien pero, en un libro de obra, en un contrato, pa ti escribir bien es darle informe a los niños, enseñarle pa [sic] ti pa [sic] que escribir bien es que te equivoquí [sic] de diente, o le dís [sic] otro remedio, no sé.» (Profesora de Construcción Civil)

\section{1.c. Relación con las diferentes esferas de actividad académica}

Otro aspecto relevante tiene relación con la problematización del lugar que esta práctica ocupa en el quehacer de los diferentes actores del contexto académico. Es así como, a medida que los profesores se adentraron en la discusión propuesta, se puso en tensión la relevancia que este tipo de escritura 
tiene para los estudiantes y egresados de carreras más profesionalizantes. También se discutió si la escritura académica sería una actividad relegada únicamente a la comunicación entre académicos:

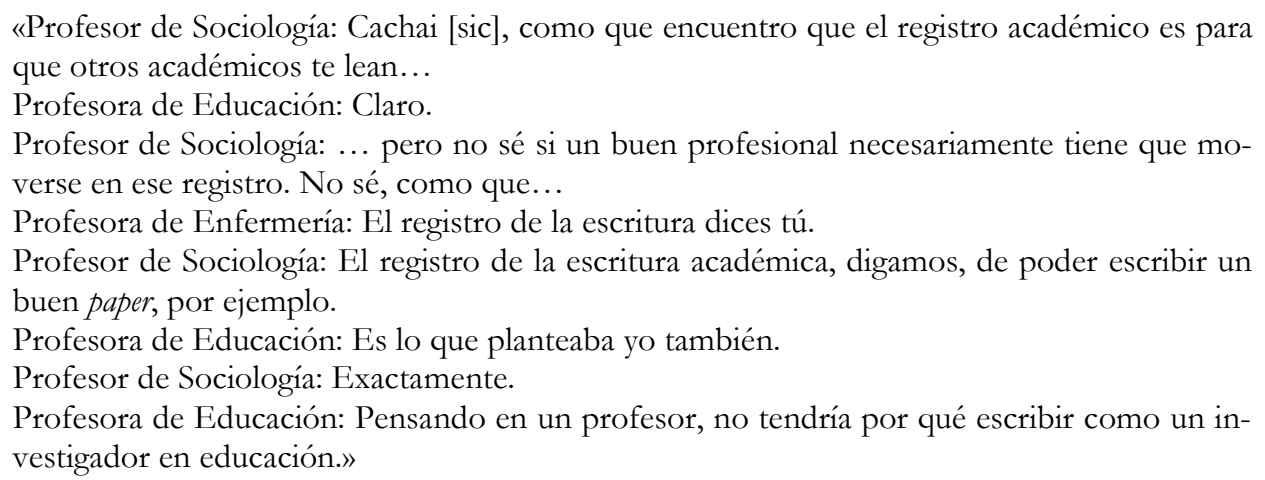

Es así como se vislumbra una cierta diferenciación entre las actividades de escritura propias de los académicos, las de los profesionales egresados de una formación universitaria, así como también de los estudiantes de la educación superior. Pese a lo anterior, los límites no resultan definidos y tampoco se genera una sistematización de las posibles diferencias existentes. Incluso se hace referencia a que en las prácticas docentes esta indefinición estaría operando de forma negativa para los estudiantes, al exigirles desempeñarse escrituralmente de acuerdo a las necesidades y estándares del quehacer académico (en un sentido profesional) y no de acuerdo a las necesidades formativas de estos:

\begin{abstract}
«Cuando haga un informe a unos padres no va a escribir un artículo ISI, ah, el que tiene que saber dar un diagnóstico de un estudiante, un reporte de su desempeño, proponer estrategias de mejora, en fin. Entonces son otras-donde está el foco, y entonces muchas veces como que estamos transmitiéndoles desde un punto de vista como nuestras propias necesidades sobre qué nos están exigiendo que escribamos. Y se nos desdibuja un poco qué es lo que necesitan los alumnos...» (Profesora de Educación)
\end{abstract}

\title{
1.d. Relevancia de la escritura para el desempeño académico y el desempeño profesional
}

Como último aspecto emergente, se destaca la gran importancia que los docentes otorgan al rol que ocupa la escritura en cuanto al quehacer disciplinar. Por una parte, la escritura mediaría y sería parte indisociable de las prácticas diciplinares, tal como indica un profesor de Odontología: «O sea, en el fondo, para sacar un muela, tú tenís [sic] que tener una ficha bien hecha, sino no la sacas». Por otra parte, los docentes plantean la escritura como medio y herramienta de pensamiento, sobre todo en el contexto de la formación universitaria: «la escritura es la única manera de transmitir lo que piensan» (profesora de Arte).

Pese a lo último, los docentes reportan que esta actividad tendría más relevancia en el contexto del futuro ejercicio profesional que en el proceso de formación académica de los estudiantes. Es así como sus acciones de apoyo al proceso de enculturación disciplinar y discursiva a los estudiantes tienden a enfocarse más en los desafíos que deberán enfrentar los alumnos una vez egresados que en aquellos que deben sortear a lo largo de su trayectoria formativa:

«No, les digo, el tema de la escritura en la carrera es superimportante porque afuera ustedes tienen que escribir contratos, que escribir en el libro de obra, y escribir un currículum vitae... Entonces, tienen que escribir, si no es porque... ¿`se fijan? Entonces eso trato yo de llevarlo harto como siendo súper pesá [sic], diciéndoles, no es de pesá [sic], y no es porque el curso está marcado que te vamos a bajar por ortografía, es porque de verdad afuera es importante.» (Profesora de Construcción Civil)

\subsection{Representaciones sociales sobre la enseñanza-aprendizaje de la escritura académica}

A continuación, se sistematizan los hallazgos relativos a las RS de los docentes en relación a la enseñanza-aprendizaje de la escritura académica. Tal como se indicó anteriormente, el segundo estímulo de los grupos de discusión consistió en que los profesores, como conjunto, indicaran qué se requiere 
para aprender a escribir académicamente. En este contexto, lo expresado por los docentes relevó siete RS relativas a la enseñanza-aprendizaje de esta habilidad, las cuales, en gran parte, están basadas en sus experiencias en el aula y como escritores académicos exitosos.

\section{2.a. Se aprende a escribir académicamente a partir de la lectura}

Una primera representación acerca de cómo los estudiantes aprenden a escribir académicamente es que lo anterior se logra a partir de la lectura. Es así como los docentes plantean que el que los estudiantes interactúen con otros textos impactaría en que ellos desarrollen una escritura adecuada: «Bueno, a veces por ejemplo, los alumnos dicen mira la verdad es que yo no sé cómo hacerlo, qué se yo, a través de buenas lecturas van cambiando también. Mucha lectura, mucha lectura.» (Profesora de Educación)

Para algunos docentes, lo anterior se lograría al entrar en contacto con textos de índole técnica, ya que así los alumnos adquirirían un mayor manejo del tecnolecto y del campo específico del conocimiento en el que están insertándose: «Que lean, que tú les pasí [sic] papers de casos aplicados y al momento de estar leyendo los paper van a adquirir el lenguaje técnico, que es lo que ellos necesitan. Conceptos, definiciones, no sé.» (Profesora de Construcción Civil)

También los estudiantes mejorarían su escritura a partir de un ejercicio constante de lectura de textos de cualquier índole y campo: «Leer, o sea, para mí es fundamental. La lectura de cualquier índole. Eh, periódicos, o leer libros de literatura, eh, estar leyendo.» (Profesora de Construcción Civil).

\section{2.b. Se aprende a escribir académicamente a partir del ejemplo del docente como escritor académico}

Una segunda RS relativa a la enseñanza-aprendizaje de la escritura académica es el desarrollo de habilidad a partir del ejemplo de los profesores, específicamente de su figura como escritores académicos. En este contexto, un profesor de Teología indica la importancia de dar ejemplo como docente: «Claro, entonces, el ejemplo de que yo escribo, de que soy riguroso... o sea, yo tengo que ser el primero.»

El rol del profesor como figura ejemplificadora cobraría especial relevancia: «Porque todos yo creo que recordamos profesores que nos han marcado en uno y otro sentido, o sea yo creo que igual el modelaje, o cuando hay pasión por algo eso agarra.» (Profesora de Enfermería)

\section{2.c. Relevancia de la motivación de los estudiantes por aprender a escribir académicamente}

Un tercer núcleo de esta línea de RS estaría vinculado a la relevancia que la motivación y el interés por escribir adecuadamente tiene para que los estudiantes aprendan a escribir en el contexto académico. La comprensión, por parte los alumnos, de la relevancia que conlleva una escritura adecuada en el contexto de las disciplinas y profesiones los ayudaría a poner mayor atención y énfasis en la calidad de sus productos escritos: «Cuando dice qué necesitan, lo primero es ver la importancia... que reconozcan lo importante de esto, o sea, como el primer paso. Porque si uno parte con una actividad y no ha reforzado o fundamentado suficientemente bien para ellos...» (Profesora de Enfermería).

De cara a evidenciar esta importancia para los estudiantes, los profesores buscan hacer explícito el rol que ocupa la escritura en el quehacer de la formación académica y en el del futuro laboral de sus alumnos:

«Constructores de afuera que ya llevan años trabajando y los invitamos a que den una charla como motivacional. Y ellos solos dicen: oigan, chicos, el tema de la escritura, cuando a mí me llega el currículo de alguien con faltas ortográficas ya no quedó clasificado, no, no, lo saco del 
archivo y no le doy la pega. Y ahí los alumnos cachan que uno es pesá [sic] con la ortografía porque en la vida real afuera...» (Profesora de Construcción Civil)

\title{
2.d. Relevancia de la evaluación de la escritura académica para su enseñanza-aprendizaje
}

Otro aspecto revelado por los docentes es la importancia de la evaluación y calificación de la escritura en los textos de los estudiantes. En este contexto resultaría relevante la incorporación de dimensiones relativas a esta en los instrumentos de evaluación de los trabajos escritos, de forma que tome relevancia en el contexto del curso:

«Profesora de Educación: Yo creo que las evaluaciones tienen que ir dirigidas, como lo que estábamos comentando en este rato, tienen que ir dirigidas a incorporar en la rúbrica de evaluación elementos clave que sean como...

Profesor de Odontología: La forma.

Profesora de Educación: ... formadores y castigadores también. O sea, yo le voy a bajar puntaje si comete tres a que cometa veinte. Yo creo que eso sí... incluso hasta en su nota. Profesora de Construcción Civil: Pero también puede ser que los bonifiquí [sic].»

En cuanto a la evaluación, también tendría relevancia el medio por el que se realiza. De esta manera, se considera que propiciar las respuestas a preguntas abiertas o la escritura de trabajos, informes, ensayos, entre otros, apoyaría el desarrollo de la escritura académica por parte de los estudiantes:

\begin{abstract}
«Preguntas que te estimulen hacia una reflexión crítica sobre el tema. Y cuesta. Después van aprendiendo pero no es la costumbre porque, como en la mayoría de las pruebas son de alternativas por la cantidad de alumnos, las de desarrollo implican harto tiempo de corrección, entonces no están acostumbrados. Yo creo que eso es lo que hace más significativo el aprendizaje... O sea tiene que ver con la... no sé si es estrategia o qué, también con la evaluación, o sea cómo se enseñan y cómo se evalúan estos aspectos.» (Profesora de Enfermería)
\end{abstract}

\section{2.e. Entrega de feedback como propiciador de la enseñanza-aprendizaje de la escritura académica}

La enseñanza-aprendizaje de la escritura académica también estaría relacionada con recibir feedback constante de la escritura en el contexto de la producción textual de los estudiantes en su trayectoria formativa. La indicación de las debilidades y fortalezas en este aspecto, por parte de docentes y ayudantes, entregaría pistas significativas a los alumnos sobre su desempeño y necesidades de mejora. Lo anterior les permitiría mejorar sus habilidades de escritura académica: «Yo digo que al menos mi feedback con ellos funciona. Y probablemente esa sea la herramienta. Que uno puede decir me va bien o no me va bien.» (Profesor de Odontología).

Con respecto al tipo de retroalimentación que debe entregarse para propiciar la enseñanza-aprendizaje, los profesores son enfáticos en que no se debe entregar la solución a los problemas o dar feedbacks meramente correctivos. Aquello que posibilitaría el que los estudiantes puedan mejorar sería la indicación de las debilidades en la escritura de modo que los alumnos se enfrenten al desafío de arreglarlo por sí mismos:

«Profesora de Enfermería: Yo antes lo hacía así. Yo yo decía, no, no me gusta así, me gusta así. Hasta que una vez lo hice de otra manera, porque al final tú como escribes, entonces vas 
quedando con todo, y lo bueno que tiene es que se marca. Ellos marcan este comentario, por ejemplo, y sale ahí la palabra...

Profesora de Educación: Yo también lo hago así.

Profesora de Enfermería: Y sabes qué, es fantástico. Porque al principio también trataba como de arreglárselo.

Profesora de Arte: No, claro.

Profesora de Enfermería: Y no po [sic], el aprendizaje.

Profesor de Sociología: Hay que mostrar.

Profesora de Enfermería: Es como dárselo hecho. Entonces ellos van a tener que buscar de nuevo cómo redactar esa frase para que se entienda.»

\section{2.f. Incorporación de instancias especificas para el desarrollo de la escritura académica en el currículum}

Otro elemento que permitiría aprender a los estudiantes a escribir académicamente sería la incorporación, a nivel curricular, de instancias específicas de desarrollo de esta habilidad. Pese a que los docentes indican que se realizan acciones en el contexto de sus cursos, consideran que estas no resultan suficientes: «Yo creo que necesitan específicamente instancias de desarrollo de la escritura porque, claro, uno dentro de la disciplina hace lo que puede pero en los contenidos del curso no está la escritura. En los contenidos del curso están los temas que vai [sic] a trabajar.» (Profesora de Arte)

En este escenario aparece como propuesta que los estudiantes cuenten con una oferta de cursos obligatorios que estén dirigidos, específicamente y por área disciplinar, al desarrollo de la escritura académica:

«Profesora de Química: Yo creo que la única opción es obligarlos a los cursos mínimos, o sea los electivos, que por obligación tengan un, por ejemplo, cada área del conocimiento-salud, humanistas, científicos, así de ciencia básica- tengan un pool muy acotado de cursos que tomar que apunten a una buena escritura...

Profesor de Teología: Claro.

Profesora de Odontología: Claro.»

\section{2.g. Se aprende a escribir académicamente con práctica}

Una séptima RS relacionada con la enseñanza-aprendizaje de la escritura académica por parte de los estudiantes es que esta se logra a partir de la práctica, pese a que hay diferentes posturas respecto al nivel de mediación del docente de dicho aprendizaje en la práctica.

Por una parte, hay profesores que consideran que una práctica guiada y mediada por el docente es lo que permite dicho aprendizaje: «En el fondo se aprende con la práctica. No hay un curso que te enseñe a traducir lo que tú dices al lenguaje normal. En el fondo, uno lo va enseñando y es más bien pragmático el tema.» (Profesor de Odontología)

Por otra parte, hay profesores que plantean que la práctica, en sí misma, podría permitir el desarrollo de la escritura académica de los estudiantes:

«Y también pienso que van adquiriendo porque quieran o no quieran, porque en todos los cursos teóricos, o sea, sobre todo en los cursos teórico prácticos, que empiezan de segundo año, enfrentan a pacientes, tiene que hacer educación por escrito y a veces formularios, eh, y los informes también que hacen de cada experiencia... entonces, también es, al final por cansancio uno va aprendiendo.» (Profesora de Enfermería)

\section{Discusión y conclusiones}

A partir de los resultados anteriormente presentados, la presente investigación de naturaleza exploratoria permitió indagar en las RS de docentes de una universidad chilena en relación a la escritura académica y su enseñanza-aprendizaje. Entre las RS encontradas a destacar se encuentra el acuerdo entre los docentes sobre la relevancia de que los estudiantes desarrollen habilidades de escritura académica y la necesidad de que exista una instancia, fuera del contexto de las cátedras que ellos tienen a cargo, de trabajo de esta herramienta. También cabe destacar ciertas RS de los docentes que develan debilidades y fortalezas en su abordaje de la enseñanza-aprendizaje de esta habilidad, en términos de 
cuánto se alejan o acercan a los hallazgos y teorías presentes en la literatura especializada en el campo de la enseñanza-aprendizaje de la escritura académica, que se ha referido al inicio de este texto. Entre las primeras se encuentran la ausencia del trabajo de la escritura desde un enfoque de la misma como proceso; la falta de claridad de los elementos estructurales de los géneros académicos; la existencia de confusión de lo que conlleva escribir académicamente en las diferentes esferas de actividad, diferenciando las acciones de escritura propias de la formación de aquellas pertenecientes al campo profesional; y la consideración de que se puede aprender a escribir académicamente a partir de la mera lectura de textos. En relación a sus fortalezas, destaca la relevancia que los profesores atribuyen a la evaluación y retroalimentación, a la práctica y a la motivación de los estudiantes por generar productos escritos de calidad para la enseñanza-aprendizaje de esta herramienta.

La relevancia de que los estudiantes desarrollen herramientas de escritura académica aparece como un consenso entre los docentes participantes, aunque dicha importancia estaría más centrada en el futuro desempeño profesional, lo que es concordante con los resultados de López y Pedraza (2012). Por otra parte, y a diferencia de los resultados de estudios como el de Ortiz (2015), en este caso los docentes sí muestran un especial interés por trabajar la escritura académica en el contexto de sus cátedras e implementan dispositivos orientados para ello. Pese a que lo anterior señala condiciones favorables para la generación de énfasis de desarrollo de esta herramienta en los cursos, resulta necesario trabajar con los docentes en torno a la comprensión de la importancia que tiene esta habilidad para que los estudiantes puedan tener una trayectoria formativa exitosa en el transcurso de sus carreras.

En relación al trabajo que realizan en el contexto de sus cátedras en pos del desarrollo de esta habilidad, los docentes consideran que no resulta suficiente por lo que debe existir una instancia específica para el desarrollo de la escritura académica en el currículum. Dicho resultado es concordante con los resultados de otros estudios como el de López y Pedraza (2012), aunque hay un elemento novedoso ya que en este caso los profesores sí se consideran responsables del desarrollo de esta habilidad por parte de sus alumnos. En este punto cabe destacar otro punto diferenciador con los resultados de otras investigaciones como las de López y Pedraza (2012), ya que los docentes indican la importancia de que estos cursos o instancias paralelas a sus cátedras tengan un enfoque disciplinar y no generalista en cuanto al trabajo de la escritura académica, lo que concuerda con su caracterización de la alta situacionalidad de la escritura según los diferentes contextos disciplinares.

Pese a lo anterior, las estrategias de enseñanza-aprendizaje que los profesores implementan en sus cursos resultarían afectadas por la falta de claridad que estos demuestran respecto a las características formales y estructurales de los textos que se escriben en el contexto académico-disciplinar y a la no incorporación de una perspectiva de la escritura como proceso (planificación, textualización y revisión iterativas), aspecto ampliamente desarrollado en la literatura especializada al respecto (Kellog, 1994; Hayes \& Flowers, 1980; Flower et al. 1986; Graham \& Perin, 2007; Didactext, 2003). La ausencia de este enfoque en el abordaje de la enseñanza-aprendizaje de la escritura académica por parte de los profesores también se ha constatado en otros estudios como los de Ortiz (2015) y Natale (2011).

Una tercera debilidad en el abordaje de la enseñanza-aprendizaje de esta herramienta por parte de los docentes se encontraría en su RS relativa a que se aprende a escribir académicamente a partir de la lectura de textos. Pese a que el conocer, a través de esta lectura, las expectativas de desempeño escrito propias de la comunicación académica aporta al desarrollo de esta habilidad, no basta la mera exposición a estos modelos sino que se requiere su análisis (Graham \& Perin, 2007). Por último, otro aspecto que podría obstaculizar la labor pedagógica de los docentes en torno a la enseñanza-aprendizaje de esta habilidad es la inexistencia en sus RS de una distinción conceptual y práctica clara de qué es lo que los estudiantes deben enfrentar, en términos de desafíos y productos relativos a la escritura académica, en función de su formación en el campo y las herramientas que necesitan desarrollar para desempeñarse como profesionales de cada área. Esta confusión podría estar generando un traslape de las exigencias de producción que ellos tienen como académicos productores de conocimiento disciplinar especializado a aquello que los alumnos deben generar en el contexto de su formación, lo que podría no concordar con el nivel de dominio del campo con que los alumnos cuentan.

En relación a las fortalezas que presentan los docentes para la enseñanza-aprendizaje de la escritura académica en el contexto de sus cátedras, destaca la relevancia que los profesores atribuyen a la motivación de los estudiantes por generar productos escritos de calidad, lo que se relaciona con la evidencia de que para alcanzar un nivel de experticia en cuanto a las habilidades de escritura avanzadas 
se requiere un alto nivel de motivación por parte del aprendiz (Alexander, 1997). También es el caso del rol que los docentes otorgan a la práctica para el aprendizaje de esta habilidad. Según estos, a través de ella los estudiantes mejorarían su nivel de escritura académica, lo que coincide con lo indicado por Kellogg \& Whiteford (2009). Por otra parte, los docentes también indican que la enseñanzaaprendizaje de la escritura académica se relacionaría con el evaluar y retroalimentar los procesos y productos de escritura de los estudiantes, estrategias que se encuentran documentadas como propiciadoras de la mejora de la escritura de los alumnos (Sommers, 1982).

A partir de lo desarrollado hasta este punto, cabe recoger tres aspectos fundamentales basados en los resultados. En primer lugar, dada la íntima relación entre RS y el quehacer docente, es recomendable, antes de realizar la implementación de una política institucional abocada a que los profesores integren estrategias de enseñanza-aprendizaje destinadas al desarrollo de esta habilidad, trabajar en pos de la generación de un sentido común compartido de qué es la escritura académica, de cuál es el sentido de trabajarla, de qué es lo que los alumnos necesitan desarrollar en cada ámbito de su trayectoria formativa y de cuál es la relevancia del rol del docente disciplinar al respecto. En segundo lugar, para el diseño de cualquier intervención es necesario indagar en el contexto disciplinar específico en el que se busca impactar. Es así como las implementaciones institucionales deben considerar la alta situacionalidad de la escritura académica, ya que las RS encontradas develan el problema de considerar la escritura académica como una actividad unitaria y transversal a todos los campos del saber. Por último, de modo de que los docentes puedan mediar de modo más eficiente la enseñanza-aprendizaje de esta habilidad, se les debe acompañar para la adquisición de una mayor conciencia y claridad de las características formales y de estructura de los géneros que son propias de su contexto disciplinar, así como también trabajar con ellos en torno al conocimiento de la importancia y de estrategias para trabajar la escritura como proceso a partir de la incorporación de acciones de planificación, textualización y revisión iterativas de los productos textuales que se producen en sus cátedras.

Para terminar, cabe indicar las limitaciones del presente estudio, que deben ser consideradas tanto para la evaluación de sus alcances como para futuras líneas de investigación. Por una parte, no se logró trabajar el muestreo como una instancia constante (Flick, 2012) desarrollado hasta lograr la saturación de las categorías trabajadas de modo de posibilitar la comprensión del fenómeno estudiado a partir de los datos recogidos. Por otra parte, se presenta el problema de la autoselección de los docentes para participar en el estudio. Podría hipotetizarse que los profesores que participaron tienen un especial interés en el tema, lo que puede sesgar el alcance de los resultados. Es por lo anterior que, para tener una idea más precisa de qué tan difundidas están las RS encontradas en esta investigación, cabe realizar estudios más amplios que consideren otras comunidades disciplinares y otras técnicas muestrales.

\section{Referencias}

Alexander, P. A. (1997). Mapping the multidimensional nature of domain learning: The interplay of cognitive, motivational, and strategic forces. En M. L. Maehr, \& P. R. Pintrich (Eds.), Advances in motivation and achievement, 10 (pp. 213-250). Greenwich, CT: JAI Press.

Bardin, L. (1996). El análisis de contenido. Madrid, España: Akal.

Canales, M. (2006). El grupo de discusión y el grupo focal. En M. Canales (Ed.), Metodologías de investigación social. Introducción a los oficios (pp. 265-287). Santiago, Chile: LOM.

Carter, M. (2007). Ways of Knowing, Doing, and Writing in the Disciplines. College Composition and Communication, 58(3), pp. 385-418.

Castelló, M., \& Mateos, M. (2015). Las representaciones de profesores y estudiantes sobre la escritura académica en las universidades españolas. Cultura y Educación, 27(3), pp. 477-503. doi: 10.1080/11356405.2015.1072357

Castorina, J. A. (2010). Los modelos de explicación para las novedades del desarrollo. Revista de Psicologia, 11, pp. 13-25.

Castorina, J. A., \& Kaplan, C. (1995). La inteligencia escolarizada. Reflexiones sobre el sentido común de los maestros y su eficacia simbólica. Revista del IICE, IV (7), pp. 105-114.

Castorina, J. A., \& Kaplan, C. (1997). Representaciones sociales y trayectorias educativas. Una relación problemática. Educaçao \& Realidade, 22(2), pp. 187-202. 
Castorina, J. A., \& Kaplan, C. (2003). Las representaciones sociales: problemas teóricos y desafíos educativos. En J. A. Castorina (Ed.), Las representaciones sociales: problemas teóricos y conocimientos infantiles (pp. 9-26). España: Gedisa.

Grinnell, R. M., \& Unrau, Y. A. (Eds.). (2005). Social work: Research and evaluation. Quantitative and qualitative approaches (7a ed., pp. 403-420). New York, NY: Oxford University Press.

Corcelles, M., Oliva, A., Castelló, M. \& Milian, M. (2015). Escribir en la universidad: ¿nos entendemos? Cultura y Educación, 27(3), pp. 534-568. doi: 10.1080/11356405.2015.1072359

Curry, W., \& Hager, E. (1987). Assessing general education. Trenton State College. En D. F. Halpern (Ed.), Student outcomes assessment: What institutions stand to gain (pp. 57-65). San Francisco, CA: JosseyBass. doi: 10.1002/he.36919875909

Creswell, J. W. (2007). Qualitative Inquiry and Research Design. Choosing among five approaches. California, CA: Sage Publications.

Didactext. (2003). Modelo sociocognitivo, pragmalingüístico y didáctico para la producción de textos escritos. Didáctica (Lengua y Literatura), 15, pp. 77-104.

Duveen, G., \& Lloyd, B. (2003). Las representaciones sociales como una perspectiva de la psicología social. En J. A. Castorina (Ed.), Las representaciones sociales: problemas teóricos y conocimientos infantiles (pp. 29-39). España: Gedisa.

Ericsson, A. (2006). The influence of experience and deliberate practice on the development of superior expert performance. En K. A. Ericsson, N. Charness, P. J. Feltovich, \& R. R. Hoffman (Eds.), The Cambridge bandbook of expertise and expert performance (pp. 683-703). Cambridge, UK: Cambridge University Press. doi: 10.1017/CBO9780511816796.038

Farr, R., \& Moscovici, S. (1984). Social representations. Cambridge, London: Cambridge University Press.

Flick, U. (2012). Introducción a la investigación cualitativa. Madrid, España: Morata.

Flower, L. S., Hayes, J. R., Carey, L., Schriver, K., \& Stratman, J. (1986). Detection, diagnosis, and the strategies of revision. College Composition and Communication, 37, pp. 16-55. doi: 10.2307/357381

Graham, S., \& Perin, D. (2007). A meta-analysis of writing instruction for adolescent students. Journal of Educational Psychology, 99, pp. 445-476. doi: 10.1037/0022-0663.99.3.445

Grinell, R. (1997). Social work research \& evaluation: Quantitative and qualitative approaches (5a ed.). Illinois, IL: Peacock Publishers.

Hayes, J. R., \& Flower, L. S. (1980). Identifying the organization of writing processes. En L. W. Gregg \& E. R. Steinberg (Eds.), Cognitive processes in writing (pp. 3-30). Hillsdale, NJ: Erlbaum.

Hernández, G. (2012). Teorías implícitas de escritura en estudiantes pertenecientes a dos comunidades académicas distintas. Perfiles Educativos, 34(136), pp. 42-60.

Hernández, R., Fernández, C., \& Baptista, P. (2010). Metodología de la investigación. (5a ed.). México: McGraw Hill.

Kellogg, R. (1994). The psychology of writing. New York, NY: Oxford University Press.

Kellogg, R. \& Whiteford, A. (2009). Training advanced writing skills: the case of deliberate practice. Educational Psychologist, 44(4), pp. 250-266. doi: 10.1080/00461520903213600

Larraín, A., \& Haye, A. (2014). A dialogical conception of concepts. Theory \& Psychology, 24(4), pp. 459478. doi: $10.1177 / 0959354314538546$

López, K., \& Pedraza, C. (2012). Cambios en las representaciones y en las prácticas de docentes universitarios sobre la escritura a partir de una estrategia colaborativa de formación en Alfabetización Académica (Tesis de maestría). Universidad del Valle, Cali, Colombia.

Marková, I. (1996). Towards an epistemology of social representations. Journal for the Theory of Social Behaviour, 26, pp. 177-196. doi: 10.1111/j.1468-5914.1996.tb00528.x

Marincovich, J., \& Salazar, J. (2011). Representaciones sociales acerca del proceso de Escritura Académica: el caso de la tesis en una Licenciatura en Historia. Estudios Pedagógicos, 37(1), pp. 85-104. doi: 10.4067/S0718-07052011000100005

Marincovich, J., Velásquez, M., Córdova, A., \& Cid, C. (2016). Academic literacy and genres in university learning communities. Ilha do Desterro, 69(3), pp. 95-113. doi: 10.5007/2175-8026.2016v69n3p95

McCutchen, D. (1988). Functional automaticity in children's writing: A problem of meta-cognitive control. Written Communication, 5, pp. 306-324. doi: 10.1177/0741088388005003003

Miño, P., Hugo, E., \& Sánchez, V. (2012). Informe de diagnóstico del proyecto «Proyecto de implementación de comunidades WAC en las Unidades Académicas de la UC». Facultad de Letras, Pontificia Universidad Católica de Chile. 
Miras, M. (2000). La escritura reflexiva: aprender a escribir y aprender acerca de lo que se escribe. Infancia y aprendizaje, 89, pp. 65-80. doi: 10.1174/021037000760088099

Moscovici, S. (2003). Des représentations collectives aux représentations sociales: éléments pour une histoire. En D. Jodelet (Ed.) Les représentations sociales (7a ed., pp. 79-103). Paris, France: PUF. doi: 10.3917/puf.jodel.2003.01.0079

Natale, L. (2011). La enseñanza y el aprendizaje de los géneros discursivos en la educación superior. Interacciones entre las representaciones de los profesores universitarios sobre la escritura académica, las valoraciones acerca de los estudiantes ya la elección de los géneros solicitados en las materias. Texturas, 11(10), pp. 87-109. doi: 10.14409/texturas.v1i11.2900

Ortiz, E. (2011). La escritura académica universitaria: estado del arte. Íkala, 16(28), pp. 17-41.

Ortiz, E. (2015). Actividades y tareas de escritura académica en el contexto universitario (pregrado). Íkala, 20(3), pp. 343-358. doi: 10.17533/udea.ikala.v20n3a05

Roux, R. (2008). Prácticas de alfabetización académica: lo que los estudiantes dicen de la lectura y la escritura en la Universidad. En Narváez, Elizabeth \& Cadena, Sonia. Los desafíos de la lectura y la escritura en la educación superior: caminos posibles (pp. 1-489). Cali, Colombia: Universidad Autónoma de Occidente.

Sommers, N. (1982). Responding to student writing. College Composition and Communication, 33(2), pp. 148-156. doi: $10.2307 / 357622$

Stagnaro, D., \& Chosco, C. (2013). Discordancias entre expectativas docentes y representaciones de los estudiantes en torno a las tareas de escritura académica: diagnóstico y algunas propuestas de intervención. En L. Natale (Coord.), El semillero de la escritura (pp. 95-128). Buenos Aires: Universidad Nacional de General Sarmiento.

Swales, J. (1990). Genre Analysis: English in Academic and Research Settings. Boston, MA: Cambridge UP.

Wagner, W., Duveen, G., Farr, R., Jovchelovitch, S., Lorenzi-Cioldi, F., Marková, I., \& Rose, D. (1999). Theory and method of social representations. Asian Journal of Social Psychology, 2, pp. 95-125. doi: 10.1111/1467-839X.00028.

White, E. M. (1989). Developing successful college writing programs. San Francisco, CA: Jossey-Bass. 